\title{
Quantum spin compass models in two-dimensional electronic topological metasurfaces
}

\author{
F. O. Nigmatulin $\odot,{ }^{1}$ I. A. Shelykh, ${ }^{2,1}$ and I. V. Iorsh ${ }^{1}$ \\ ${ }^{1}$ Faculty of Physics, ITMO University, St. Petersburg 197101, Russia \\ ${ }^{2}$ Science Institute, University of Iceland IS-107, Reykjavik, Iceland
}

(Received 21 June 2021; accepted 3 September 2021; published 7 October 2021)

\begin{abstract}
We consider a metasurface consisting of a square lattice of cylindrical antidots in a two-dimensional topological insulator (2DTI). Each antidot supports a degenerate Kramer's pair of eigenstates formed by the helical topological edge states. We show that the on-site Coulomb repulsion leads to the onset of the Mott insulator phase in the system in a certain range of experimentally relevant parameters. Intrinsic strong spin-orbit coupling characteristic for the 2DTI supports a rich class of the emerging low-energy spin Hamiltonians that can be emulated in the considered system, which makes it an appealing solid-state platform for quantum simulations of strongly correlated electron systems.
\end{abstract}

DOI: 10.1103/PhysRevResearch.3.043016

\section{INTRODUCTION}

Spin-lattice models are ubiquitous in theoretical physics. Besides their natural applications for the description of the behavior of magnetic systems, a variety of the condensed-matter problems related to high-temperature superconductivity [1], thin superfluid films [2], quantum Hall bilayers [3], and nonlinear optical lattices [4] allow mapping into spin-lattice Hamiltonians. An interesting subclass of such models is represented by Compass models (CM), for which the characteristic feature is direction-dependent spin-spin interaction [5]. The first model of this type was introduced back in the 1982 [6] to describe the interplay between the Jahn-Teller effect and magnetization dynamics. Since then, CMs have been applied for modeling of emergent phenomena in a variety of strongly correlated systems such as high-temperature superconductors [7,8], vacancy centers networks [9], colossal magnetoresistance manganites [10], and materials supporting spin-liquid phases [11]. One of the most prominent examples is the Kitaev model [12] employed extensively in the rapidly developing field of topological quantum computation.

For any spin model, it is highly desirable to find a material platform that allows flexible control over its effective parameters [13]. While it has been argued that certain quantum CMs can be emulated with the use of cold atoms in optical lattices $[14,15]$, corresponding solid-state platforms are still yet to appear. Here we demonstrate that a metasurface formed by a square lattice of antidots in two-dimensional topological insulators (2DTI) is an attractive alternative for this.

The 2DTIs are materials that have both a $2 \mathrm{D}$ bulk energy gap (like ordinary insulators) and 1D gapless conducting

Published by the American Physical Society under the terms of the Creative Commons Attribution 4.0 International license. Further distribution of this work must maintain attribution to the author(s) and the published article's title, journal citation, and DOI. edges [16-18] protected by time-reversal symmetry and characterized by the spin-momentum locking, which means that at a specific boundary the direction of the propagation of an edge state is uniquely defined by electron spin projection. Naturally, for the closed boundaries, the energy of an edge state is quantized, and for the case of rotational symmetry, the corresponding eigenstates are characterized by specific projections of the orbital and spin angular momentum. Such topological resonators have been actively studied recently in topological photonic systems [19-21], but can be realized as well for electrons, an example being an antidot (ring shape aperture) in 2DTI. The electronic spectrum of such a system was obtained in Refs. [22,23] and associated quantum impurity models have been considered in a number of follow-up works [24,25].

In the current paper, we show that a square lattice of antidots in 2DTI emulates a quantum spin CM with additional spin-orbit interaction of the Dzyaloshinskii-Moriya (DM) type. The parameters of the model can be flexibly tuned by change of the geometry of the lattice (antidot size and interdot distance), which can be routinely achieved within state-of-the-art fabrication techniques. The proposed system can thus serve as a solid-state quantum simulator of a wide class of quantum CMs with possible applications ranging from emulation of correlated electron materials to topological quantum computation.

The geometry of the system we consider is shown schematically in Fig. 1. We take an example of the $\mathrm{CdTe} / \mathrm{HgTe} / \mathrm{CdTe}$ quantum well [18], since this is currently the most common of 2DTI where topologically protected edge states has been observed experimentally [26], but other material platforms are also possible.

The model Hamiltonian of a $\mathrm{CdTe} / \mathrm{HgTe} / \mathrm{CdTe}$ quantum well is represented by a $4 \times 4$ block-diagonal matrix, which consists of the blocks related to each other by time-reversal symmetry operation [18], $H=\operatorname{diag}\left[h(k), h^{*}(-k)\right]$, where

$$
h(k)=\left(C-D k^{2}\right)+\left(M-B k^{2}\right) \sigma_{z}+A \sigma \cdot \mathbf{k} .
$$




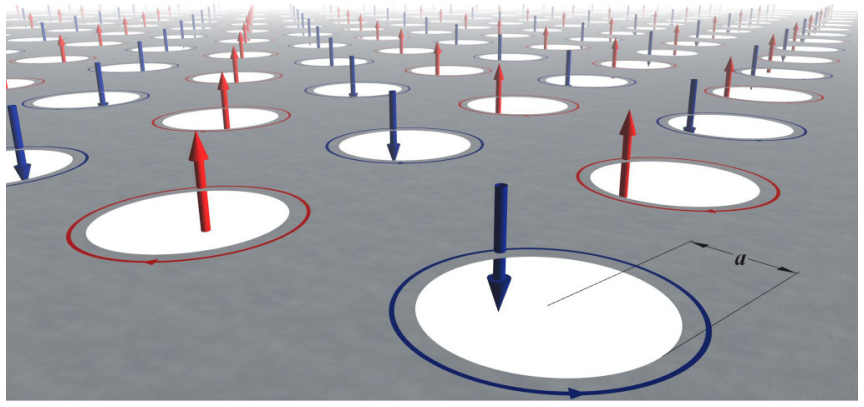

FIG. 1. A 2D array of antidots (holes) in 2DTI. Blue (dark) and red (light) arrows show topologically protected counterpropagating edge states with opposite spin directions.

The parameters entering this expression are determined by the geometry of the QW. In what follows, we take $A=375 \mathrm{mev} \mathrm{nm}, B=-1.12 \mathrm{ev} \mathrm{n^{2 }}, D=0, C=0, M=$ $-10 \mathrm{mev}$ [22]. The parameter $M$ (Dirac mass), defined by a thickness of a QW, is of special importance, as only the case $M<0$ corresponds to the topologically nontrivial regime.

The eigenvalues and eigenvectors of an individual axially symmetric antidot can be found with use of the following ansatz for a four-spinor:

$$
\psi=\frac{e^{i m \theta}}{\sqrt{2 \pi}}\left(\begin{array}{c}
\chi_{1}^{m}(r) e^{i \theta / 2} \\
\chi_{2}^{m}(r) e^{-i \theta / 2}
\end{array}\right)
$$

where $\theta$ is an angular coordinate, $\chi_{1,2}^{m}(r)$ are radial parts of a wave function, and quantum number $m=$ $\pm 1 / 2, \pm 3 / 2, \pm 5 / 2, \ldots$ gives $\mathrm{z}$ component of the total angular momentum $j_{z}$ commuting with the Hamiltonian. Using this substitution we get the radial part of a wave function in terms of the Macdonald functions $K_{m \pm 1 / 2}$. Applying the Dirichlet boundary condition at the antidot edge $\left.\chi_{1,2}(r)\right|_{r=a}=$ 0 , we get a secular equation defining the eigenenergies and eigenfunctions.

The spectrum of a single antidot as a function of an antidot radius $a$ is shown in Fig. 2. As one can see, the spectrum is symmetric with respect to the gap center (zero energy). For

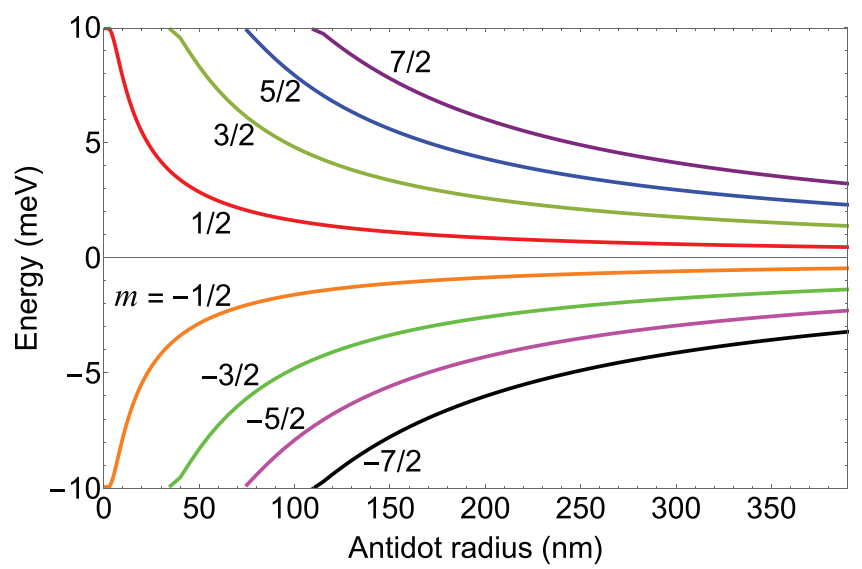

FIG. 2. Energies of in-gap edge states circulating around an antidot as functions of the antidot radius $a ; m$ is the quantum number defining $\mathrm{z}$ projection of the total angular momentum of the circulating edge states.
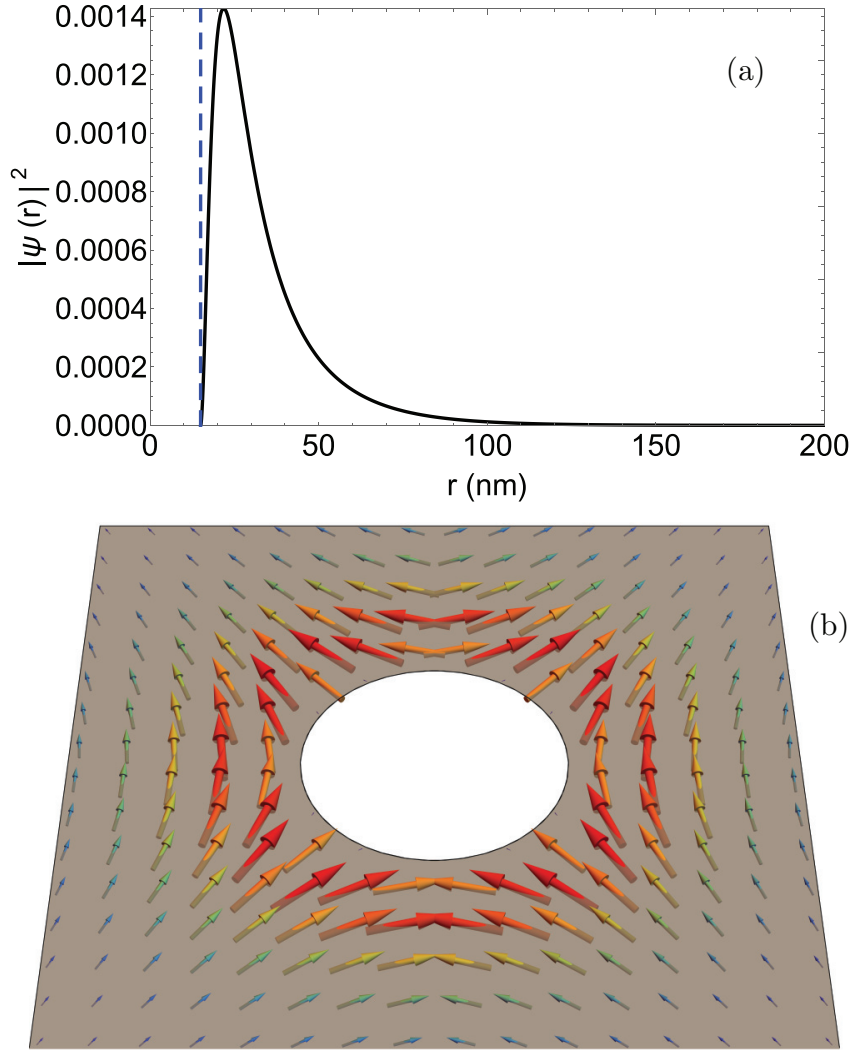

FIG. 3. (a) The dependence of the $|\psi(r)|^{2}$ of the edge state of an antidot with the radius $a=15 \mathrm{~nm}$ vs distance from the center of the antidot, $|m|=1 / 2$. (b) Spin density of the edge state near a single antidot.

small radii there exist only two bound states corresponding to $m= \pm 1 / 2$ with energies approaching the gap edges as one decreases the radius. At larger radii the states corresponding to larger $|m|$ appear. In what follows, we consider the radius $a=15 \mathrm{~nm}$, which corresponds to the case of a single pair of the bound states.

It should be stressed that each of the eigenenergies corresponds to the Kramer's doublet, representing a mixture of purely orbital degenerate states [27] and degenerate spin states. We introduce the pseudospin index $\sigma=(\uparrow, \downarrow)$ to label the partners of the doublet. Their wave functions $\psi$ and $\psi^{\prime}$ are related to each other by the time-reversal operator $\mathcal{T}$ : $\psi^{\prime}=\mathcal{T} \psi=i \sigma_{y} \psi^{*}$.

In Figs. 3(a) and 3(b) we plot the probability distribution functions and corresponding spin-density profile for the $m=$ $1 / 2$ eigenstate for $a=15 \mathrm{~nm}$. It can be seen that the wave function is localized at the scale of several $a$. Moreover, the spin distribution of the state is highly nonisotropic, which is a consequence of the spin-orbit coupling.

In the situation, when apertures are placed reasonably close to each other, the wave functions of the states localized at each of them overlap, and electrons can thus tunnel between antidots. If one neglects the doublet corresponding to higher energy (upper line in Fig. 2), the system can be described in terms of a tight-binding Hamiltonian of the Hubbard type, 
which for a square lattice of antidots reads:

$$
\begin{aligned}
H= & -\sum_{\langle i, j\rangle}\left(c_{i \sigma}^{+} t_{i j}^{\sigma \sigma^{\prime}} c_{j \sigma^{\prime}}+\text { H.C. }\right)+U \sum_{i}\left(n_{i \uparrow}-\frac{1}{2}\right) \\
& \times\left(n_{i \downarrow}-\frac{1}{2}\right),
\end{aligned}
$$

where $c_{i \sigma}$ is the annihilation operator for the state with specific pseudospin projection localized at site $i$ of the lattice. The first term corresponds to the tunneling between the sites, while the last term describes on-site Coulomb repulsion.

Since we account for nearest-neighbor hopping only, there exist only two inequivalent tunneling matrices $t_{i, i+\hat{x}}^{\sigma \sigma^{\prime}}, t_{i, i+\hat{y}}^{\sigma \sigma^{\prime}}$ corresponding to hoppings along orthogonal lattice translation vectors, which read:

$$
t_{i, i+\hat{e}}^{\sigma \sigma^{\prime}}=\epsilon \int d^{2} \mathbf{r} \psi_{\sigma}^{H}(\mathbf{r}) \psi_{\sigma^{\prime}}(\mathbf{r}+R \hat{e}),
$$

where $R$ is the distance between the antidot centers, $\epsilon$ is the bound state energy, and $\hat{e}=[x, y]$. Substituting the expression for $\psi_{\uparrow}$ from Eq. (2) and recalling that $\psi_{\downarrow}=\mathcal{T} \psi_{\uparrow}$, we notice that $t_{i, i+\hat{e}}^{\uparrow \uparrow}=t_{i, i+\hat{e}}^{\downarrow \downarrow}$ and $t_{i, i+\hat{e}}^{\uparrow \downarrow}=-\left(t_{i, i+\hat{e}}^{\downarrow \uparrow}\right)^{*}$. Moreover, since $\chi_{1}, \chi_{2}$ are real functions, the diagonal elements $t^{\sigma \sigma}$ are real. Also, the absolute value of tunneling amplitudes should depend only on the distance between antidots and not on the orientation of the antidot pair. These general considerations allow to parametrize the tunneling matrix as

$$
\hat{t}_{i, i+\hat{e}}=t\left(\begin{array}{cc}
\cos \alpha & e^{i \phi_{\hat{e}}} \sin \alpha \\
-e^{i \phi_{\hat{e}}} \sin \alpha & \cos \alpha,
\end{array}\right),
$$

where $t$ and $\alpha$ are real numbers, which depend on the antidot radius and intersite distance, and phase $\phi_{\hat{e}}$ depends on the hopping direction. Numerical integration shows that $\phi_{x}=0$ and $\phi_{y}=\pi / 2$. The dependence of the tunneling amplitude $t$ and phase $\alpha$ versus intersite distance $R$ for $a=15 \mathrm{~nm}$ is shown in Fig. 4(a). As expected, the tunneling amplitude decays exponentially with the inersite distance. Interestingly, the angle $\alpha$ reaches the value of $\pi / 4$, which corresponds to the case of the equivalency of spin conservative and spin-flip tunnelings, at some finite value of $R$.

The Coulomb interaction energy can be estimated as:

$$
U=\int V\left(\mathbf{r}_{1}, \mathbf{r}_{2}\right) \rho_{\uparrow}\left(\mathbf{r}_{1}\right) \rho_{\downarrow}\left(\mathbf{r}_{2}\right) d \mathbf{r}_{1} d \mathbf{r}_{2},
$$

where $\rho_{\uparrow \downarrow}(\mathbf{r})=\psi_{\uparrow \downarrow}^{\dagger}(\mathbf{r}) \psi_{\uparrow \downarrow}(\mathbf{r})$ is an electron density for the corresponding pseudospin projection, and $V$ is the interaction potential, which in principle should include both static and dynamical screening. We will neglect the latter, since edge states lie in the band gap of the bulk material where there is vanishing density of the free electrons. As to the static screening, it was considered in Ref. [28] where it has been shown that the the effect of the image potential can be neglected for moderate dielectric contrasts and simple expression for the potential $V=e^{2} /\left(\varepsilon\left|\mathbf{r}_{1}-\mathbf{r}_{2}\right|\right)$, where $\varepsilon \approx 10$ is the static dielectric constant of HgTe and can be safely used.

In Fig. 4(b) we plot the dependence of $U$ on the antidot radius $a$. Its nonmonotonic behavior can be attributed to the fact that for small antidots, the eigenfunctions are weakly localized in the radial direction because of the approaching of
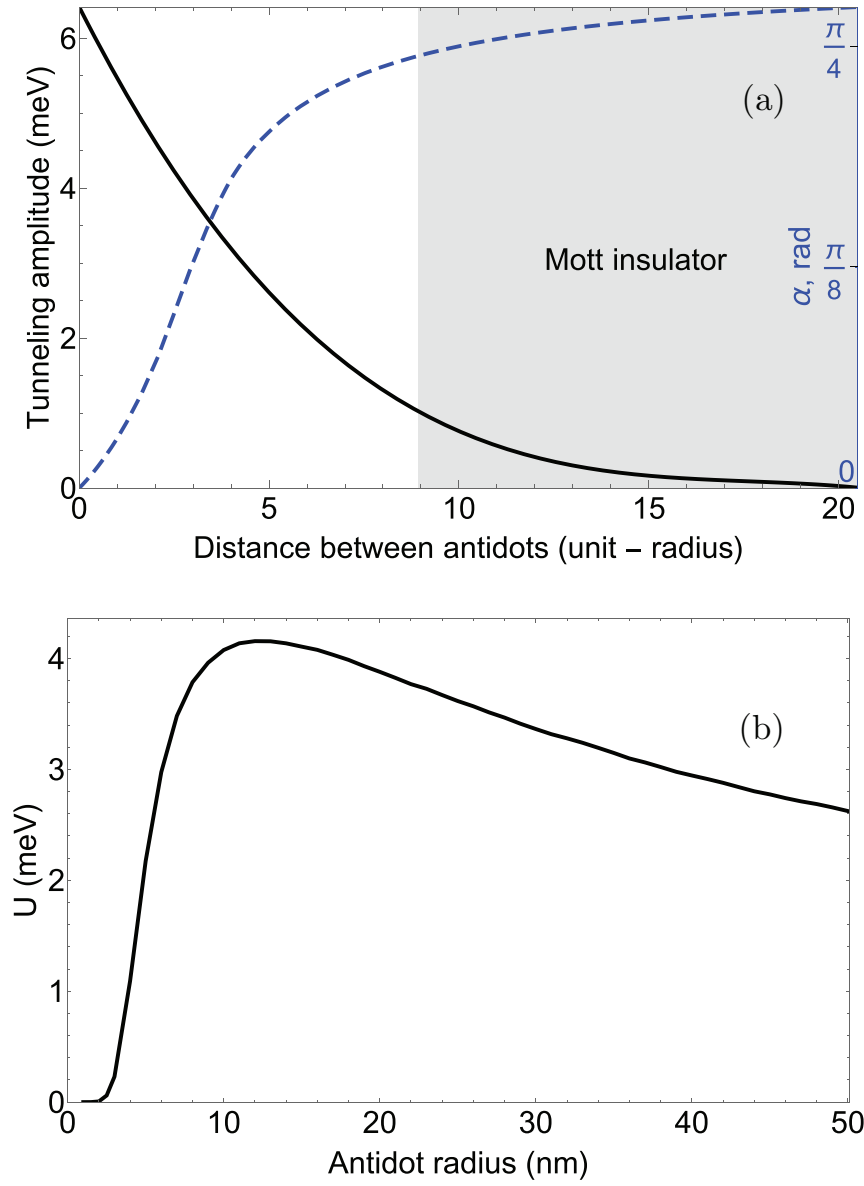

FIG. 4. (a) The dependence of the tunneling amplitude (black curve) and $\alpha(\beta)$ parameter (dashed blue curve) vs distance between centers of antidots $\mathrm{R}$ (in units of the dot radius $a$, taken to be $15 \mathrm{~nm}$ ). The gray area of the plot denotes Mott insulating regime of the system at half filling. (b) The dependence of the on-site interaction energy vs radius of the antidot

the corresponding energies to the band-gap edge. At the same time, for large antidots, wave function becomes delocalized along its periphery of the radius $\approx 2 a$. The interplay between these two effects defines the radius at which $U$ becomes maximal. For the considered parameters, $U=U_{\max } \approx 4 \mathrm{mev}$ is achieved at $a \approx 13 \mathrm{~nm}$.

Let us consider the situation when the Fermi energy in the system is tuned in such a way that one has exactly one electron per each dot (regime of half filling). It is well known that in this situation, the tunneling between the neighboring sites can be completely blocked by the interaction. This regime corresponds to the so-called spin-orbit coupled Mott insulator and is achieved when $U>4 t$ [29], which in our case corresponds to the distances between the antidots $R>10 a$.

Our geometry resembles some previously proposed ones, where Mott insulators were realized with arrays of semiconductor quantum dots [30-32]. However, there is one crucial difference, namely, strong spin-orbit coupling inherent for the TIs. It gives an additional twist to our model, which enables to emulate a much wider class of the accessible low-energy Hamiltonians. 
By employing the standard Schrieffer-Wolff transformation and excluding the states with two electrons sitting on the same sites, we can map the low-energy sector of the original Hamiltonian 3 into the following spin-lattice model:

$$
H_{\mathrm{eff}}=J \sum_{i}\left[S_{i}^{a} R_{a b}^{x}(2 \alpha) S_{i+\hat{x}}^{b}+S_{i}^{a} R_{a b}^{y}(2 \beta) S_{i+\hat{y}}^{b}\right],
$$

where exchange constant $J=4 t^{2} / U>0, a, b=1,2,3$ denotes components of the pseudospin operator; $\hat{x}, \hat{y}$ are basis vectors along corresponding axes; and $R^{x}(2 \alpha), R^{y}(2 \beta)$ are $\mathrm{SO}(3)$ rotation matrices around $x$ and $y$ axes, respectively. The states with pseudospins $S_{z}= \pm 1 / 2$ correspond to the occupations of the partners of the Kramers doublet, other states being their linear combinations.

For the case of $\alpha=\pi / 4$, which is reached for the intersite distance $\approx 10 a$, we can rewrite the Hamiltonian as

$$
H_{\mathrm{eff}}=J \sum_{i} \sum_{\hat{e}=\hat{x}, \hat{y}} S_{i}^{e} S_{i+\hat{e}}^{e}+\hat{e} \cdot\left[\mathbf{S}_{i} \times \mathbf{S}_{i+\hat{e}}\right],
$$

where the first term corresponds to the so-called $90^{\circ}$ spin $\mathrm{CM}$ and the second term to the conventional DzyaloshinskiiMoriya interaction (DMI).

The Hamiltonian in Eq. (8) is essentially a quantum spin CM with added DMI interaction. Pure spin CMs are usually characterized by the highly degenerate ground states, which sometimes allow for the dimensionality reduction and even the exact solution such as in the case of the Kitaev model, corresponding to the honeycomb lattice [5]. While for the case of the square lattice, the DMI interaction is likely to lift the ground-state degeneracy, it will be instructive to consider the structures with geometrical frustration, such as Lieb or Kagome lattices, and to explore the interplay between the geometrical frustration and the strong spin-orbit coupling. Moreover, inclusion of the edge states characterized by a larger value of $m$ opens the access to the multiband Hubbard model and to the multiband Mott insulators with strong spinorbit interaction.

In the classical limit, the Hamiltonian (8) is characterized by the spiral wave ground states [15]. At the same time, quantum fluctuations can substantially modify the groundstate properties of the system. Specifically, in Ref. [33], the 1D analog of Hamiltonian (8) was analyzed, where the spins are aligned along the $x$ axis. It has been shown using exact diagonalization and renormalization group methods that the spiral long-range order characterized by the order parameter,

$$
C_{h}=\frac{1}{4 N} \sum_{i=1}^{N}\left\langle S_{i}^{z} S_{i+1}^{y}-S_{i}^{y} S_{i+1}^{z}\right\rangle,
$$

is destroyed and only local order is preserved.

To probe the onset of different collective phases experimentally, one can resort to the measurement of the zero-bias conductance [32], which can be performed for different filling factors, controlled by the gate voltage. To spot quantum phase transitions, one would need cryogenic temperatures $T$ satisfying the condition $T \approx 0.01 t \approx 100 \mathrm{mK}$ in order to preserve the correlations from being washed away by thermal fluctuations $[34,35]$. This temperature range can be routinely achieved in the modern dilution cryostats.

In conclusion, we have proposed an experimentally viable quantum simulator of a spin-orbit coupled Mott insulator based on an array of antidots in the 2DTI. If the antidot size is sufficiently small, the low-energy behavior of the system can be described by the quantum spin CM with DMI. The alternative lattice geometries supporting the geometrical frustration would give access to an even richer class of the available spin models, which could be of particular interest in the domains of quantum simulation and quantum computation.

\section{ACKNOWLEDGMENTS}

The authors acknowledge the support of Russian Science Foundation Grant No. 20-12-00224.
[1] P. A. Lee, N. Nagaosa, and X.-G. Wen, Doping a Mott insulator: Physics of high-temperature superconductivity, Rev. Mod. Phys. 78, 17 (2006).

[2] D. R. Nelson, Superfluidity and the two dimensional $x y$ model, Phys. Rep. 49, 255 (1979).

[3] O. Kyriienko, K. Wierschem, P. Sengupta, and I. A. Shelykh, Quantum hall bilayer as pseudospin magnet, Europhys. Lett. 109, 57003 (2015).

[4] K. P. Kalinin, A. Amo, J. Bloch, and N. G. Berloff, Polariton XY-Ising machine, Nanophotonics 9, 4127 (2020).

[5] Z. Nussinov and J. van den Brink, Compass models: Theory and physical motivations, Rev. Mod. Phys. 87, 1 (2015).

[6] K. I. Kugel and D. I. Khomsk, The Jahn-Teller effect and magnetism: transition metal compounds, Sov. Phys. Usp. 25, 231 (1982)

[7] K. Kuroki, S. Onari, R. Arita, H. Usui, Y. Tanaka, H. Kontani, and H. Aoki, Unconventional Pairing Originating from the Disconnected Fermi Surfaces of Superconducting lafeaso ${ }_{1-x} f_{x}$, Phys. Rev. Lett. 101, 087004 (2008).
[8] F. Krüger, S. Kumar, J. Zaanen, and J. van den Brink, Spinorbital frustrations and anomalous metallic state in iron-pnictide superconductors, Phys. Rev. B 79, 054504 (2009).

[9] F. Trousselet, A. M. Oleś, and P. Horsch, Magnetic properties of nanoscale compass-heisenberg planar clusters, Phys. Rev. B 86, 134412 (2012)

[10] Y. Tokura and Y. Tomioka, Colossal magnetoresistive manganites, J. Magn. Magn. Mater. 200, 1 (1999).

[11] S.-H. Baek, S.-H. Do, K.-Y. Choi, Y. S. Kwon, A. U. B. Wolter, S. Nishimoto, J. van den Brink, and B. Büchner, Evidence for a Field-Induced Quantum Spin Liquid in $\alpha-\mathrm{RuCl}_{3}$, Phys. Rev. Lett. 119, 037201 (2017).

[12] A. Yu Kitaev, Fault-tolerant quantum computation by anyons, Ann. Phys. 303, 2 (2003).

[13] T. S. Cubitt, A. Montanaro, and S. Piddock, Universal quantum hamiltonians, Proc. Natl. Acad. Sci. USA 115, 9497 (2018).

[14] C. Wu, Orbital Ordering and Frustration of P-Band Mott Insulators, Phys. Rev. Lett. 100, 200406 (2008). 
[15] J. Radić, A. Di Ciolo, K. Sun, and V. Galitski, exotic Quantum Spin Models in Spin-Orbit-Coupled Mott Insulators, Phys. Rev. Lett. 109, 085303 (2012).

[16] M. Z. Hasan and C. L. Kane, Colloquium: Topological insulators, Rev. Mod. Phys. 82, 3045 (2010).

[17] C. L. Kane, Chapter 1 - topological band theory and the $\mathcal{Z}_{2}$ invariant, in Topological Insulators, Contemporary Concepts of Condensed Matter Science, Vol. 6, edited by Marcel Franz and Laurens Molenkamp (Elsevier, Amsterdam, 2013), pp. 3-34.

[18] B. A. Bernevig and T. L. Hughes, Topological Insulators and Topological Superconductors (Princeton Univ. Press, Princeton, 2013).

[19] S. Barik, A. Karasahin, S. Mittal, E. Waks, and M. Hafezi, Chiral quantum optics using a topological resonator, Phys. Rev. B 101, 205303 (2020).

[20] M. Jalali Mehrabad, A. P. Foster, R. Dost, E. Clarke, P. K. Patil, I. Farrer, J. Heffernan, M. S. Skolnick, and L. R. Wilson, A semiconductor topological photonic ring resonator, Appl. Phys. Lett. 116, 061102 (2020).

[21] M. J. Mehrabad, A. P. Foster, R. Dost, E. Clarke, P. K. Patil, A. M. Fox, M. S. Skolnick, and L. R. Wilson, Chiral topological photonics with an embedded quantum emitter, Optica 7, 1690 (2020).

[22] P. Michetti and P. Recher, Bound states and persistent currents in topological insulator rings, Phys. Rev. B 83, 125420 (2011).

[23] W.-Y. Shan, J. Lu, H.-Z. Lu, and S.-Q. Shen, Vacancy-induced bound states in topological insulators, Phys. Rev. B 84, 035307 (2011).

[24] H.-F. Lü, H.-Z. Lu, S.-Q. Shen, and T.-K. Ng, Quantum impurity in the bulk of a topological insulator, Phys. Rev. B 87, 195122 (2013).
[25] X. Xin and D. Zhou, Kondo effect in a topological insulator quantum dot, Phys. Rev. B 91, 165120 (2015).

[26] M. König, H. Buhmann, L. W. Molenkamp, T. Hughes, C.-X. Liu, X.-L. Qi, and S.-C. Zhang, The quantum spin hall effect: Theory and experiment, J. Phys. Soc. Jpn. 77, 031007 (2008).

[27] J. van den Brink and D. Khomskii, Double Exchange Via Degenerate Orbitals, Phys. Rev. Lett. 82, 1016 (1999).

[28] S. T. Cui, Electrostatic potential in cylindrical dielectric media using the image charge method, Mol. Phys. 104, 2993 (2006).

[29] G. Jackeli and G. Khaliullin, Mott Insulators in the Strong SpinOrbit Coupling Limit: From Heisenberg to a Quantum Compass and Kitaev Models, Phys. Rev. Lett. 102, 017205 (2009).

[30] C. A. Stafford and S. Das Sarma, Collective Coulomb Blockade in an Array of Quantum Dots: A Mott-Hubbard Approach, Phys. Rev. Lett. 72, 3590 (1994).

[31] R. Ugajin, Mott metal-insulator transition driven by an external electric field in coupled quantum dot arrays and its application to field effect devices, J. Appl. Phys. 76, 2833 (1994).

[32] T. Byrnes, N. Y. Kim, K. Kusudo, and Y. Yamamoto, Quantum simulation of fermi-hubbard models in semiconductor quantum-dot arrays, Phys. Rev. B 78, 075320 (2008).

[33] R. Jafari, M. Kargarian, A. Langari, and M. Siahatgar, Phase diagram and entanglement of the ising model with Dzyaloshinskii-Moriya interaction, Phys. Rev. B 78, 214414 (2008).

[34] W. Hofstetter, J. I. Cirac, P. Zoller, E. Demler, and M. D. Lukin, High-Temperature Superfluidity of Fermionic Atoms in Optical Lattices, Phys. Rev. Lett. 89, 220407 (2002).

[35] T. A. Maier, M. Jarrell, T. C. Schulthess, P. R. C. Kent, and J. B. White, Systematic Study of $d$-Wave Superconductivity in the 2D Repulsive Hubbard Model, Phys. Rev. Lett. 95, 237001 (2005). 